\title{
Cultura y valores en el proceso de discriminación de las personas transgénero
}

Inés Arístegui ${ }^{1}$

Universidad de Palermo - Fundación Huésped

Artículo científico. Material original autorizado para su primera publicación en Journal de Ciencias Sociales, Revista Académica de la Facultad de Ciencias Sociales de la Universidad de Palermo.

Recibido: 1-10-2015

Aceptado: 23-10-2015

Resumen

La Psicología Social y Cultural estudia la interrelación entre los aspectos culturales y los procesos psicológicos, convirtiéndose en un enfoque apropiado para explorar cómo la cultura de un país y los valores individuales de sus ciudadanos se relacionan con los procesos de estigma y discriminación vividos por la población transgénero. A tal fin, el presente ensayo primero describe brevemente las dimensiones culturales de Hofstede y la Teoría de los Valores Básicos de Schwartz. Seguidamente, analiza la relación entre cultura y prejuicio en general y el conflicto intergrupal con la población transgénero en particular. Se describe cómo la transgresión a las creencias y valores culturalmente compartidos, así como a las normas establecidas acerca de lo masculino y femenino, es el principal atributo estigmatizante de la población transgénero. Así, se presenta cómo la Ley de Identidad de Género, en tanto cambio en las normas que justifican las acciones de los individuos, promueve cambios en las creencias y patrones de conducta de los individuos, que depararán en una mejora de la calidad de vida de las personas transgénero y en una disminución de las situaciones de estigma y discriminación. Asimismo, se describe cómo la promoción y la educación en valores que faciliten el respeto y la aceptación de "los otros", durante el proceso de socialización familiar y escolar, servirían como herramientas para contrarrestar el estigma. Del mismo surge la necesidad de generar políticas educativas que promuevan valores asociados a la apertura al cambio y la auto-trascendencia ya que tienden a promover la búsqueda por lo novedoso, el juicio independiente así como el respeto por la igualdad y el bienestar de los otros.

\footnotetext{
${ }^{1}$ Investigadora adjunta en el Centro de Investigación en Psicología de la Universidad de Palermo. Coordinadora del Área de Investigación Social de Fundación Huésped. Está realizando su tesis de Doctorado en Psicología. Mail: ines_aristegui@yahoo.com
} 
Palabras clave: cultura, valores, discriminación, personas transgénero

\section{Abstract}

Social and Cultural Psychology studies the relationship between culture and psychological processes and becomes an appropriate approach to explore how a country's culture and individuals' values are related to the processes of stigma and discrimination of transgender people. Thus, this essay firstly describes Hofstede's cultural dimensions and Schwartz's Theory of Values. Then, an analysis of the relationship between culture and prejudice as well as the intergroup conflict with the transgender people is presented. It is described as the transgression of culturally shared beliefs and values about the masculine and the feminine and the established norms in relation to it, are the main aspects that stigmatize transgender people. The recent Gender Identity Law, might change the institutional norms that justify individuals' actions, promoting changes in beliefs and patterns of behavior, and thus, generating improvements in transgender people's quality of life and a decrease in the situations of stigma and discrimination. This work also describes how to promote and educate in values that facilitate the respect and acceptance of others, serving as tools to counter stigma. It is conclude the need to generate educational policies that promote values associated with openness to change and self-transcendence as values that promote the search for novelty, independent judgment and respect for equality and other's wellbeing.

Keywords: culture, values, discrimination, transgender people 
El conocimiento público de las personas transgénero y sus problemáticas se ha incrementado sustancialmente a través del arte - libros, películas y programas de televisión (American Psychological Association, APA, 2009). El término transgénero es un concepto abarcativo que describe a aquellas personas que construyen una identidad de género (sentimientos, actitudes, comportamientos, vestimenta) diferente de la que le fue asignada al nacer en función de su sexo biológico, como ser, como ser transexuales, travestis e intersex. Académicamente, la investigación con esta población se ha centrado en el campo de la salud pública - $\mathrm{VIH} /$ sida y evaluaciones pre intervenciones médicas- y otras disciplinas como la sociología y los estudios de género (APA, 2009). Sin embargo, son escasos los estudios desde el campo de la psicología. Como la Psicología Social y Cultural tiene como objeto de estudio la interrelación entre los aspectos culturales y los procesos psicológicos, entendiéndola como una relación bidireccional en la que ambos están implicados, siendo a veces causa o efecto de lo que acontece en la realidad (Zubieta, 2008; Zubieta, Fernández y Sosa, 2012), esta disciplina se presenta como un enfoque apropiado para analizar uno de los fenómenos sociales más fuertemente vividos por la población transgénero: el estigma y la discriminación.

En Argentina, el interés por la población transgénero se ha vuelto más relevante desde la aprobación, en mayo de 2012, de la Ley Nro. 26.743 de Identidad de Género (Boletín Oficial, 2012) que garantiza el libre desarrollo de las personas conforme a su identidad de género, corresponda o no con el sexo asignado al momento de nacer. Esta ley garantiza el cambio de nombre en sus documentos de identidad así como el acceso a una salud integral. A diferencia de otros países con leyes similares, en Argentina no es requisito una evaluación psicodiagnóstica ni una autorización judicial o administrativa previa a realizar estos cambios. El espíritu detrás de esta ley fue garantizar los derechos humanos y civiles de las personas transgénero para mejorar así su bienestar y calidad de vida en general (Fundación Huésped, FH, 2014). En particular, se espera que esta ley, en su calidad de política pública, contribuya a disminuir las situaciones de discriminación en los diferentes ámbitos de sus vidas (Arístegui \& Vazquez, 2013; FH, 2014).

Para analizar y comprender los procesos políticos que podrían potencialmente ocurrir a partir de la Ley de Identidad de Género, en línea con lo propuesto por Staerkle y Doise (2014), es necesario tener en cuenta una perspectiva más social que conecte las explicaciones de nivel individual con análisis de la dinámica social como las normas, creencias, valores e ideologías que guían y dan sentido al comportamiento individual. Así, el presente trabajo tiene como objeto explorar cómo la cultura de un país y los valores individuales de sus ciudadanos se relacionan con los procesos de estigma y discriminación vividos por la población transgénero. A tal fin, primero se presentará una descripción de los conceptos de cultura y de la teoría de valores, para posteriormente articularlo con los procesos de estigmatización y discriminación de las personas transgénero. 


\section{Cultura y Valores}

Según Zubieta (2008), la cultura ha sido definida como un conjunto de patrones de conducta compartidos por un grupo de individuos que tienen una historia y estructura social común. Estos conocimientos compartidos son valorados y sirven de normas que guían el accionar de los individuos. Así, la cultura puede considerarse desde una visión institucional, que conlleva sanciones y refuerzos de determinadas conductas, o desde una visión psicológica, que refiere a la internalización que las personas hacen de determinados valores, actitudes y creencias que orientan su comportamiento. Según Páez y Zubieta (2004), Triandis (1994) definió a principio de los '90 los componentes de la cultura como: creencias (cómo se designa y evalúa); roles (conductas proscritas según posición en la estructura social); normas (reglas y expectativas que regulan comportamientos y emociones); y valores (principios que evalúan lo que es deseable). Cabe destacar que esta visión subjetiva de la cultura refiere a las estructuras de significado compartidas que no son estáticas y por tanto pueden ser promotoras de cambios (Páez \& Zubieta, 2004).

Por su parte, Hofstede, Hofstede \& Minkov (2010) define la cultura como la programación mental colectiva, una construcción cuyo núcleo son los valores y que diferencia a los miembros de un grupo de personas de otros grupos. Esos valores se adquieren a través del proceso de socialización fundamentalmente en los ámbitos familiar, vecinal y escolar -durante los primeros años de vida- y posteriormente, a través de otros grupos sociales tales como organizaciones recreativas, políticas, estudiantiles o laborales. Como resultado, existen tres niveles de programación mental: individual (heredado y aprendido), colectivo (aprendido) y universal (heredado).

De este modo, Hofstede et al. (2010) promueve el análisis por país y relaciona las diferencias encontradas entre las culturas nacionales con los dilemas básicos de las sociedades postulados por Inkeles y Levinson (1969, citado en Hofstede et al., 2010) acerca de la relación con la autoridad, la concepción de sí mismo (relación individuo-grupo y diferencias hombre-mujer) y el enfrentamiento del conflicto, control de la agresión y expresión de las emociones. Hoftede et al. (2010) evalúa las diferencias entre naciones a través de las siguientes dimensiones culturales: Distancia de Poder (medida en que los miembros menos poderosos de organizaciones o instituciones aceptan y esperan la distribución desigual del poder), Individualismo vs. Colectivismo (grado en que las personas se integran en los grupos), Evitación de la Incertidumbre (tolerancia de una sociedad a la ambigüedad e incertidumbre) y Masculinidad vs. Femineidad (distribución de roles emocionales entre los géneros).

Como los seres humanos tienen una personalidad individual, pertenecen a determinadas organizaciones y viven en sociedades nacionales, lo que ellos sienten, hacen y piensan depende de la interrelación en estos 
tres niveles (Hofstede et al., 2010). Es por ello que cualquier investigación en Ciencias Sociales en general, y sobre situaciones de prejuicio y discriminación en particular, debe considerar los valores como un factor explicativo de los objetivos que se proponga.

A fines del siglo XX toma fuerza el estudio de los valores, considerando la independencia e interdependencia del sujeto con su propia cultura, destacándose como perspectiva estándar la Teoría General de los Valores Humanos de Schwartz (Páez \& Zubieta, 2004; Schwartz, 2009). Schwartz (1992) define a los valores como metas motivacionales que dirigen la acción de forma relativamente estable, en función de lo que los grupos o la persona definen como deseable y significante para sus vidas. Los valores, en tanto creencias no objetivas que trascienden las acciones y situaciones específicas y se utilizan como criterios para seleccionar o evaluar acciones, políticas, hechos y personas, se ordenan según la importancia relativa de un valor con relación a otro, y se construyen en normas de evaluación y justificación de la acción. Para Schwartz (2009) los valores emergen como respuestas que dan los grupos o las personas para hacer frente a las diferentes necesidades de su existencia como organismos biológicos, a la coordinación de la interacción social y al logro del bienestar y supervivencia de los grupos. Son motivos sociales que se adquieren a través del proceso de socialización y por tanto son consonantes al grupo cultural de pertenencia del individuo.

Schwartz (1992) identificó diez categorías de valores definidos según los tipos motivacionales que se representan como meta y representados por una estructura circular que da cuenta de su importancia de acuerdo con el orden que ocupan y su posición con respecto a otros valores. Estos configuran relaciones dinámicas que pueden entrar en conflicto o ser compatibles entre ellas: 1) auto-dirección (pensamiento independiente, explorar, acción-elección); 2) estimulación (novedad y desafío en la vida); 3) hedonismo (gratificación sensual para uno mismo), 4) logro (éxito personal por demostración de competencia), 5) poder (estatus social, control de personas y recursos), 6) seguridad (estabilidad en la sociedad, las relaciones y con uno mismo), 7) conformidad (restricción de acciones e impulsos que puedan alterar o dañar a los demás y violar expectativas sociales), 8) tradición (respeto y compromiso de las costumbres de una cultura o religión), 9) benevolencia (preservar y mejorar el bienestar de aquellos con quienes uno está en contacto personal) y 10) universalismo (entendimiento, reconocimiento, valoración, tolerancia y protección para el bienestar de todas las personas y la naturaleza).

La Teoría de Schwartz (Zubieta, 2008) postula que existe un nivel básico en el que los valores forman un continuum de motivaciones relacionadas y se organizan en dos dimensiones ortogonales bipolares que subyacen a todas las acciones de los individuos. La primera dimensión contrasta los valores de Apertura al cambio (autodirección y estimulación, valores que promueven la independencia de juicio y acción y favorecen el cambio) con los de Conservación (seguridad, conformidad y tradición, valores que preservan la 
tradición, protegen la estabilidad y enfatizan la auto-represión). En la segunda dimensión, se oponen los valores de Autopromoción (poder y logro, valores que hacen hincapié en el éxito personal y el dominio sobre otros) con los de Autotrascendencia (universalismo y benevolencia, valores que enfatizan la aceptación de la igualdad de los otros y la preocupación por su bienestar). Schwartz (1992) plantea que las acciones que los individuos emprenden para realizar cada tipo de valor tienen consecuencias psicológicas, prácticas y sociales que pueden ser compatibles o entrar en conflicto con la realización de otro tipo de valores. Por ejemplo, al intentar mantener valores tradicionales se podría entrar en conflicto con la búsqueda de estimulación, la novedad o el desafío.

Fontaine, Poortinga, Delbeke y Schwartz (2008) proponen una nueva forma de agrupar los valores, según estén dirigidos a la expresión de los intereses y características personales o centrados en la persona (autodirección, estimulación, hedonismo, logro y poder); o regulen las relaciones con los otros y sus efectos, es decir, centrados en lo social (universalismo, benevolencia, tradición, conformidad y seguridad). Simultáneamente, realiza otra agrupación en: valores de crecimiento, dirigidos a la auto-expansión sin preocupación (autodirección, universalismo, benevolencia, estimulación y hedonismo) vs. valores de protección, que hacen hincapié en la autoprotección con preocupación (seguridad, poder, logro, conformidad y tradición).

Los valores individuales pueden ser producto de la herencia cultural o de la experiencia personal, mientras que los culturales representan prioridades sociales a través de ideas compartidas acerca de lo esperado y correcto en un grupo o sociedad. No obstante, ambos están relacionados a nivel conceptual y son interdependientes (Páez y Zubieta, 2004). Son varias las investigaciones que dan cuenta de la asociación entre las dimensiones culturales propuestas por Hofstede y la Teoría de Valores Humanos de Schwartz y (Páez y Zubieta, 2004; Zubieta, 2008).

\section{Estereotipos, prejuicio y los procesos de estigma y discriminación}

Fueron el mismo Schwartz y su colega Struch (1989) quienes analizaron la teoría de valores humanos con las creencias acerca del prejuicio, observando que las diferencias percibidas con relación a un conjunto de valores es lo que media la agresión y el conflicto intergrupal. Los estereotipos han sido definidos como creencias populares sobre los atributos que caracterizan a un grupo social y sobre las que hay un acuerdo básico (Brehm, Kassin \& Fein, 2002). Estos tienen un valor funcional y adaptativo ya que ayudan a comprender el mundo de manera más simplificada, ordenada y coherente. Además de generar un ahorro de esfuerzo cognitivos frente a un mundo desconocido y novedoso, los estereotipos tienen la función de 
facilitar una identidad social, es decir, la conciencia de pertenecer a un grupo social. Esta identidad, al igual que la cultura y los valores, se adquieren de forma muy espontánea principalmente en la infancia y la juventud.

Sin embargo, debe considerarse que el estereotipo, en cuanto categorización, mantiene una estrecha relación con el conjunto de creencias y sentimientos de carácter negativo con relación a un grupo social, denominados prejuicios, y que pueden generar y perpetuar sentimientos de superioridad de un grupo frente a otro, siendo así la base de procesos de estigmatización y discriminación (Brehm et al., 2002). Goffman (1968) definió el estigma como la identificación que un grupo social crea sobre una persona, o grupo de personas, a partir de algún rasgo físico, conductual o social que se percibe como divergente del grupo. Como consecuencia, esta identificación se convierte en un atributo devaluador que degrada y rebaja a la persona que lo posee. El estigma depende en gran medida de los significados compartidos en un periodo histórico particular y un contexto cultural determinado (Miric, 2003).

Según Link y Phelan (2001), las personas distinguen y etiquetan las diferencias humanas basadas en las creencias de la cultura dominante, ligando las personas etiquetadas a estereotipos negativos o características indeseables. Estas categorías creadas sirven para lograr una separación entre "ellos" y "nosotros", a partir de la cual se producen hechos de discriminación hacia quienes no cumplen con las características del "nosotros" impuestas culturalmente. De este modo, se entiende por actos de discriminación a toda conducta que manifieste una falta de igualdad en el tratamiento otorgado a otras personas, en virtud de su pertenencia a un grupo o categoría social sobre la que existe un cierto prejuicio (Brehm et al., 2002).

En particular, el género es una de las etiquetas sociales que las personas utilizan más frecuentemente y de manera instantánea y sistemática (Zubieta et al., 2011). Los estereotipos de género son ideas construidas y reproducidas culturalmente en torno a lo que las personas piensan y perciben acerca de cómo deben ser los roles y responsabilidades de hombres y mujeres, comenzando así a hablarse de masculinidadfeminidad. En el caso de las personas transgénero, son la principal causa de discriminación social e institucional (APA, 2009; Arístegui \& Vazquez, 2013; Barrón López, Libson, \& Hiller, 2008; FH, 2012; 2014).

\section{Discriminación basada en la dimensión cultural de masculinidad-femineidad}

En nuestra sociedad, las categorías sexo y género suelen confundirse y usarse como una sola. Según la APA (2008), el sexo biológico incluye atributos físicos como cromosomas, gónadas, estructura reproductiva interna y genitales externos; la identidad de género refiere a la percepción psicológica de 
sentirse hombre o mujer y está asociada a la forma en la que las personas se comportan, interactúan y sienten acerca de sí mismas; y los roles de género, son definidos como la serie de comportamientos, actitudes y rasgos de personalidad que una sociedad, en un momento histórico determinado, designa como masculinos o femeninos (es decir, propios de los hombres o de las mujeres).

Según Zubieta et al. (2011), el género como etiqueta social muestra una influencia de manera más marcada en culturas que, en términos de Hofstede, son más masculinas y jerárquicas, como Argentina donde aún existe una dominancia del hombre sobre la mujer. Sin embargo, el modelo binomial de femenino-masculino no da cuenta de las complejidades del género y la realidad social, lo que hace mucho más complejo su análisis (Toro-Alfonso, 2010). Por ejemplo, se ha creado una versión urbana y metropolitana del hombre que cuida su imagen, perfeccionando su cuerpo, su cutis y su forma de vestir de un modo que transgrede la directriz de lo estrictamente "masculino". Asimismo, algunos grupos de adolescentes recrean la ambigüedad de género, mezclando gestos, vestimenta y comportamientos de un modo que genera incertidumbre. Lo andrógino (altos niveles en masculinidad y femineidad) convierte en moda la transgresión y la ambigüedad de género. El comportamiento basado en el género es flexible y está influido por la situación en donde la interacción tiene lugar, es decir, en el contexto.

Las personas transgénero, a diferencia de la mayoría de la población, fluyen entre los componentes de la sexualidad y de la cultura de modo distinto: como se expuso previamente, su identidad de género no coincide con el sexo asignado al nacer y sus roles de género presentan mayor variabilidad desde aquellas personas que adscriben rigurosamente a su identidad de género hasta aquellas que presentan roles y expresiones más andróginas (Helien \& Piotto, 2012). Esta transgresión a los significados, creencias y valores culturalmente compartidos, así como a las normas establecidas acerca de lo masculino y femenino, es el principal atributo estigmatizante de la población transgénero. En tanto entidad social, esta población desafía al grupo dominante quien no sabe cómo nombrar o categorizar a esta población (ej. perversos, enfermos mentales, transexuales/transgénero, "la" o "el" travesti) y se siente amenazado por este grupo que no cumple con las expectativas deseables socialmente (sus miembros muchas veces viven en la marginalidad absoluta, realizando trabajo sexual y con altos índices de violencia y consumo de sustancias).

Debido a los diversos factores culturales, socio-políticos y religiosos asociados a la sexualidad y las normas de género que históricamente han llevado a la negación de su existencia, la población transgénero se encuentra en situación de vulnerabilidad social (Barrón López et al., 2008), siendo sistemáticamente discriminada y excluida desde muy temprana edad de sus ámbitos familiares y educativos, así como del mercado laboral formal y del sistema de salud (FH, 2012; Mizock \& Mueser, 2014). Así, como la misma APA 
(2009) sostiene, trabajar sobre la problemática de la población transgénero es, en particular, trabajar sobre sus derechos humanos.

Afortunadamente, la cultura no es estática, sino que la socialización también permite la modificación de actitudes, creencias y rasgos psicológicos entre los miembros de la misma (Páez \& Zubieta, 2004; Zubieta, 2008). De este modo, modificando las normas o constricciones externas, se espera que lo mismo ocurra con los patrones de conducta. La modificación de normas culturales a nivel institucional - como fue la Ley de Identidad de Género en nuestro país garantizando derechos políticos y civiles, así como la nueva edición del Manual Diagnóstico y Estadístico de los Trastornos Mentales (DSM-5, American Psychiatric Association, 2013), despatologizando a la población transgénero - fue paralelamente generando algunos cambios a nivel psicológico y de la que se espera impulse un cambio en las creencias y patrones de conducta de las personas.

Según la investigación cuali-cuantitativa realizada por Fundación Huésped y la Asociación de Travestis, Transexuales y Transgénero de Argentina (FH, 2014), desde la implementación de la Ley de Identidad de Género, las experiencias de violencia policial y de discriminación de los ámbitos de salud y educación disminuyeron notablemente. Se observó una reducción en el abuso psicológico y físico por parte de las fuerzas de seguridad, como así también una mejor y más apropiada atención dentro del sistema sanitario y en los centros educativos. Según las percepciones de la misma población transgénero, el cambio más significativo se ha dado dentro de la misma población, quienes han podido empoderarse y modificar sus creencias acerca de sí mismas, facilitando así un cambio en sus patrones de conducta y animándose a ejercer sus derechos civiles y políticos. Por ejemplo, cambiando sus documentos de identidad, animándose a circular de día por la ciudad, reincorporándose al sistema educativo o presentándose a búsquedas laborales formales, entre otros. Sin embargo, del mismo informe se desprende que aún resta mucho camino por recorrer a fin de realizar un cambio social más fuerte hacia la aceptación de las personas transgénero. Pero, como Kendel, Devor y Strapko (1997) encontraron, a medida que los individuos conocen - tienen contacto personal - con alguna persona transgénero, las creencias negativas acerca de este grupo social viran hacia una actitud más positiva.

\section{Los valores humanos como herramientas para derribar prejuicios}

Si los valores, como núcleo fundamental de una cultura, se adquieren principalmente durante el proceso de socialización familiar, vecinal y escolar (Hofstede et al., 2010); y como Schwartz (2009) sostiene, si se modificaran los valores que se utilizan como criterios para evaluar acciones, políticas, y personas, se 
modificarían las normas que justifican las acciones de los individuos. Así, es posible pensar que la promoción y la educación en valores que faciliten el respeto y la aceptación de "los otros" servirían como herramientas para contrarrestar el estigma y la discriminación vivida por aquellas personas que no pertenecen al grupo dominante. Pero, ¿̇uáles son esos valores que se deben promover en la sociedad?

Sobre la base de la descripción de Brehm et al, (2002) con respecto a cómo desarticular prejuicios y romper con la escalada de discriminación, a partir de establecer contactos individuales con las personas y no darles un trato como entidad grupal; y a partir de las dimensiones de valores propuestas por el mismo Schwartz y sus colegas (ver Zubieta, 2008 para revisión), podría pensarse que el foco debería estar en aquellos valores asociados a la apertura al cambio y la auto-trascendencia, ya que tienden a promover la búsqueda por lo novedoso, el juicio independiente así como el respeto por la igualdad y el bienestar de los otros. Como Struch y Schwartz (1989) mencionan, cuanto mayor es el nivel de apertura al contacto con el exo-grupo ("ellos"), mayor es la importancia del valor universalismo y menor el énfasis en la conservación. De este modo puede pensarse que, si las personas pudiesen afrontar el desafío de conocer a una persona transgénero, hacer un juicio sobre esa persona de forma independiente a los estereotipos aprendidos, entendiendo su situación de vida como consecuencia de los procesos de estigmatización y discriminación, respetando en tanto ser humano y ciudadano su derecho a gozar de una plena ciudadanía que garantice sus derechos humanos básicos, entonces los actos de discriminación contra esta población podrían disminuir y alcanzar en tanto sociedad una cultura más armoniosa.

El contacto con minorías culturales, como la población transgénero, implica exponerse a costumbres y tradiciones diferentes que son vividas como una suerte de amenaza por aquellos que dan relevancia a la tradición (Struch \& Schwartz, 1989). La falta de familiaridad con las normas del grupo minoritario dificulta la posibilidad de establecer una relación fluida entre ambos grupos. En cierta medida, lo que ocurre con el grupo dominante es que percibe que su seguridad se ve afectada. Esto pudo observarse en los medios de comunicación cuando se hizo conocido el "caso Lulu", en el cual una madre pidió a la justicia cambiar el DNI de su hija menor de edad nacida varón, pero identificada con el género femenino (Carbajal, 2013). Durante el debate ocasionado, se habló mucho de los "valores de la familia", "lo natural", "las perversiones", "la correcta educación de un niño", entre otros, y siempre con un dejo fuerte de agresión hacia la madre, sin lograr empatía ni comprensión y mucho menos información objetiva acerca de lo que esa persona estaría pasando. En términos de género, su hijo/hija no reflejaba lo que seguramente esa misma madre también habría deseado o pensado era lo esperable culturalmente, pero en términos generales, la posición de la madre fue tomada por muchas personas como una amenaza al modelo de familia y educación de los niños. 
Considerando que los valores son consonantes al grupo cultural de pertenencia Schwartz (2009), es interesante observar cómo en otras culturas las personas transgénero tienen otro status dentro de la sociedad, como el caso de las Hijras en India, las Fa'Afaines en Samoa o de las vírgenes juradas en Albania, donde han sido consideradas un tercer género o vírgenes y donde cumplen roles y funciones claras en la bendición de nuevos nacimientos, cuidados de la familia y tareas domésticas, entre otros.

Como Hofstede et al. (2010) describen, al hablar de culturas nacionales y sus diferentes valores, debe considerarse que existen fuerzas que facilitan la integración como ser el lenguaje, los medios de comunicación, y la educación; pero también están presentes otras fuerzas que la dificultan como los grupos religiosos o las diferentes identidades étnicas. En Argentina, en un estudio con más de 900 personas residentes en diferentes ciudades (Zubieta, Fernández \& Sosa, 2012), se observó que predominan los valores relacionados a la auto-trascendencia y la apertura al cambio. En alguna medida ayuda a comprender cómo un país donde aún existe una amplia brecha entre lo esperado para hombres y mujeres (Zubieta et al., 2011), y donde predomina la religión católica que promueve valores relacionados a la conservación (conformidad y tradición), ha sido posible contar con la ley del "matrimonio igualitario" y la de identidad de género. Estos cambios en las normas institucionales se presentan como una oportunidad de cambio social para los grupos minoritarios asociados a la diversidad sexual y, como los mismos miembros de estos grupos estigmatizados han expresado, han colaborado fuertemente en reducir las situaciones de discriminación (FH, 2012; 2014).

Al comparar los valores en términos de edad (Zubieta et al., 2012), se observa que las personas más jóvenes se orientan por valores de mayor auto-promoción y apertura al cambio en comparación con los de mayor edad que se van volcando hacia valores de mayor conservación. Es por esto que la educación sobre este tipo de valores debe fortalecerse desde temprana edad, ya que es justamente la etapa donde también se internalizan culturalmente los estereotipos y prejuicios hacia ciertos grupos. Zubieta, Delfino y Fernández (2007) encontraron que aquellos estudiantes con altos niveles de dominancia social tienden a puntuar bajo en universalismo y benevolencia, dos valores necesarios para poder trabajar con la aceptación del otro perteneciente a una minoría. Asimismo, estas personas también están ideológicamente más orientadas hacia la derecha, y por tanto será más difícil para estos grupos acompañar con ciertas políticas públicas que intentan generar cambios sociales en lugar de la conservación de la tradición, viéndose así afectada su percepción de seguridad y conformidad.

Como varias organizaciones civiles y organismos políticos han planteado, el trabajo en educación hacia aquellos valores que Fontaine et al. (2008) definen como asociados al crecimiento -auto-dirección, universalismo, benevolencia, estimulación- debería ser parte de una política pública y promoverse 
formalmente en el ámbito educativo. En esta línea, hoy existen organizaciones profesionales internacionales, como la Asociación de Psicólogos Americana; la Sociedad de Psicólogos Australiana y la Asociación Mundial de Profesionales de la Salud Transgénero, que han elaborado guías para trabajar con personas transgénero; así también como iniciativas civiles y gubernamentales locales e internacionales que han elaborado guías para trabajar la diversidad sexual en las escuelas como ser, el Consejo Consultivo Asesor de Derechos y Diversidad Sexual de la Ciudad de Buenos Aires y la comisión de educación del Colectivo de Lesbianas, Gays, Transexuales y Bisexuales de Madrid (COGAM).

\section{Conclusiones}

Este trabajo tuvo como objeto contribuir a comprender la relación entre cultura y valores y los procesos de estigma y discriminación de la población transgénero, para así poder diseñar mejores programas e intervenciones o generar políticas públicas orientadas a reducir la discriminación de las personas transgénero. Las Dimensiones Culturales de Hofstede y la Teoría de los Valores Básicos de Schwartz son un importante aporte teórico para comprender esa relación entre cultura y prejuicio y el conflicto intergrupal con la población transgénero. De lo expuesto, se desprende que el continuo de los valores, ya sean individuales o culturales, requiere ser considerado en la investigación aplicada para poder comprender los ejes por los que transitan los comportamientos y actitudes de los diferentes grupos humanos frente a determinadas situaciones, la dinámica de las relaciones entre el grupo dominante y el grupo estigmatizado, como así también las posibilidades de cambio o el cambio en sí mismo.

Como se ha observado, particularmente en Argentina con las leyes aprobadas en los últimos años, las culturas evolucionan según cambia el entorno político, económico, tecnológico y social. Estos cambios son importantes antecedentes que promueven cambios en los valores que una cultura sostiene como deseables. A su vez, los valores pueden ser causa de los cambios en el comportamiento y acciones humanas, como ser los procesos de estigma y discriminación. De este modo, al considerar desde la Psicología Social y Cultural el rol de la cultura, los valores cobran una gran relevancia ya que no sólo son consecuencia sino también predictores de comportamientos, sentimientos y actitudes de grupos y/o personas. En línea con lo sugerido por Staerkle y Doise (2014), el análisis en función de categorías sociales aventajadas y desaventajadas, es un componente esencial en esta disciplina. Así, como Zubieta et al. (2012) concluyen, la intervención psicosocial requiere abandonar la visión homogénea del siglo XX para incluir la diversidad en todas sus dimensiones, buscando alcanzar no solo el bienestar personal, subjetivo y psicológico de las personas, sino el bienestar social. En el trabajo con la población transgénero, en particular, es necesario considerar la 
interdependencia individuo-sociedad, pero al mismo tiempo promover el respeto por la independencia para el logro del desarrollo tanto personal como social, y en este marco, las leyes y las políticas educativas cumplen un rol fundamental. 


\section{Referencias Bibliográficas:}

American Psychiatric Association. (2013). Diagnostic and statistical manual of mental disorders (5th ed.). Arlington, VA: American Psychiatric Publishing.

American Psychological Association (2008). Resolution on transgender and gender identity and gender expression non-discrimination. Recuperado el 18 de mayo de 2010 de http://www.apa.org/pi/lgbt/programs/transgender/index.aspx

American Psychological Association (2009). Report of the Task Force on Gender Identity and Gender Variance. Washington, DC: Author.

ARÍSTEGUI, I. \& Vazquez, M. (2013). El impacto del estigma y la discriminación en la calidad de vida de personas transgénero viviendo con VIH. Hologramática, 19(1), 5-30. Recuperado el 20 de diciembre de 2014 de http://www.cienciared.com.ar/ra/usr/3/1477/hologramatica n19pp5 30.pdf.

BARRÓN LÓPEZ, S., Libson, M., \& Hiller, R. (2008). Estudio social en hombres que tienen sexo con hombres (HSH): Relevamiento 2007. Buenos Aires: Ubatec.

Boletín Oficial de la República Argentina (mayo 2012). Ley de Identidad de Género. Año CXX, Nro 32.404. Recuperado el 20 de septiembre de 2013 de http://www1.hcdn.gov.ar/BO/boletin12/2012-05/BO24-052012leg.pdf

BREHM, S. S., Kassin, S. M., \& Fein, S. (2002). Social Psychology ( $5^{\text {th }}$ ed.).Boston, MA: Houghton Mifflin.

CARBAJAL, M. (Julio, 2013). Lo que devuelve el espejo. Diario Página 12. Recuperado el 20 de diciembre de 2014 de http://www.pagina12.com.ar/diario/sociedad/3-225462-2013-07-28.html

FONTAINE, J. R. J., Poortinga, Y. H., Delbeke, L., \& Schwartz, S. H. (2008). Structural Equivalence of the Values Domain Across Cultures: Distinguishing Sampling Fluctuations From Meaningful Variation. Journal of Cross-Cultural Psychology, 39, 345-365.

Fundación Huésped (2012). Percepciones y experiencias sobre estigma y discriminación en poblaciones trans, HSH, y usuarios de drogas. Buenos Aires: Autor. Recuperado el 20 de diciembre de 2014 de http://www.huesped.org.ar/wp-content/uploads/2012/06/Informe-Final-Percepciones.pdf

Fundación Huésped (2014). Ley de Identidad de Género y acceso al cuidado de la salud de las personas trans en Argentina. Buenos Aires: Autor. Recuperado el 20 de diciembre de 2014 de http://www.huesped.org.ar/wp-content/uploads/2014/05/OSI-informe-FINAL.pdf

GOFFMAN, E. (1968). La identidad deteriorada. Buenos Aires: Amorrortu. 
HELIEN, A. \& PIOTTO, A. (2012). Cuerpxs equivocadxs: hacia la comprensión de la diversidad sexual. Buenos Aires: Paidós.

HOFSTEDE, G., HOFSTEDE, G. J., \& MINKOV, M. (2010). Cultures and Organizations: Software of the Mind (3rd edition). New York: McGraw-Hill USA.

KENDEL, M., DEVOR, H., \& STRAPKO, N. (1997). Feminist and lesbian opinions about transsexuals. En B. Bullough, V. Bullough, \& J. Elias (Eds.), Gender blending (pp. 146-159). Amherst, NY: Prometheus.

LINK, B. \& PHELAN, J. (2001). Conceptualizing stigma. Annual Review of Sociology, 27, 363-385.

MIRIC, M. (2003). Las huellas del Tabú, aproximaciones al concepto de estigma. Paradigmas, 1, 50-62.

MIZOCK, L., \& MUESER, K. T. (2014). Employment, mental health, internalized stigma, and coping with transphobia among Transgender Individuals. Psychology of Sexual Orientation and Gender Diversity, 1(2), $146-158$.

PÁEZ, D., \& ZUBIETA, E. (2004). Cultura y Psicología Social. En D. Páez, I. Fernández, S. Ubillos, \& E. Zubieta (coords.), Psicología Social, Cultura y Educación (pp. 25-53). Madrid: Pearson- Prentice Hall.

SCHWARTZ, S. (1992). Universals in the content and structure of values: Theoretical advances and empirical tests in 20 countries. En M. Zanna (Ed.), Advances in Experimental Social Psychology (pp. 1-65). San Diego: Academic Press.

SCHWARTZ, S. H. (2009). Basic human values. Sociologie, 42, 249-288.

SCHWARTZ, S.H. y Struch, N. (1989). Values, stereotypes, and intergroup antagonism. En Daniel Bar-Tal y Graumann, C., Kruglanski, A. y Stroebe, W. (Eds), Stereotyping and prejudice. Nueva York: SpringerVerlag.

STAERKLE, C., \& DOISE, W. (2014). De la psicología social a la psicología política: el abordaje societal. En E. Zubieta, J. Valencia \& G. Delfino (coords.). Psicología Social y Política: Procesos teóricos y estudios aplicados (pp. 23-52). Buenos Aires: EUDEBA.

TORO ALFONSO, J. (2010). El cuerpo y el género trans-formados: A modo de introducción. Identidades: Revista interdisciplinaria de Estudios de las Mujeres y el Género, 8, 87-100.

ZUBIETA, E. (2008). Valores humanos y conducta social. En M. M. Casullo, Psicología Positiva (pp. 203-229). Buenos Aires: Lugar.

ZUBIETA, E., DELFINO, G. \& FERNÁNDEZ, O. (2007). Dominancia social, valores y posicionamiento ideológico en jóvenes universitarios. Psicodebate: Psicología, Cultura y Sociedad, 8, 151-169.

ZUBIETA, E., FERNÁNDEZ, O., \& SOSA, F. (2012). Bienestar, valores y variables asociadas. Boletín de Psicología. Universidad de Valencia, España. ISSN 0212-8179. 
ZUBIETA, E., BERAMENDI, M., SOSA, F., \& TORRES, A. (2011). Sexismo ambivalente, estereotipos y valores en el ámbito militar. Revista de Psicología, 29, 101-130. 